\title{
EXIT TIME FUNCTIONALS FOR INTEGER-VALUED POISSON PROCESSES
}

UDC 519.21

\author{
D. V. GUSAK
}

\begin{abstract}
The joint distribution of all exit time functionals is studied in this paper for a fixed level $x$ and integer-valued compound Poisson processes. An exact formula for the distributions of these functionals is obtained in the case of semicontinuous processes. Limit relations are obtained for the distributions of the exit time functionals for $x=0$ or as $x \rightarrow \infty$.
\end{abstract}

The distribution of exit time functionals is studied in [1]-3 for homogeneous processes with independent increments and with jumps whose distribution function is continuous. The same problem is studied in 4, 5, for integer-valued processes $\xi(t)$ defined on finite Markov chains. Moreover, limit distributions as $x \rightarrow \infty$ ( $x$ is an integer) are given in [4, 5] under the condition that $m_{1}=\mathrm{E} \xi(1) \geq 0$. We obtain an exact formula for the distribution of exit time functionals in the case of semicontinuous compound Poisson processes for both cases $m_{1} \geq 0$ and $m_{1}<0$. As a corollary of these relations we obtain an assertion on the distribution of extreme values of a semicontinuous integer-valued Poisson process. In particular, we obtain a discrete analogue of the Pollaczek-Khintchine formula for the distribution of the global maximum in the case of $m_{1}<0$.

Consider an integer-valued Poisson process $\xi(t), t \geq 0$, such that $\xi(0)=0$ and the moment generating function and cumulant are given by

$$
p_{t}(u)=\mathrm{E} u^{\xi(t)}=e^{t \psi(u)}, \quad|u|=1,
$$

and

$$
\begin{gathered}
k(u)=\lambda \sum_{k \neq 0}\left(u^{k}-1\right) p_{k}=\lambda(p(u)-1), \quad|u|=1, \lambda>0, \\
p(u)=\sum_{k \neq 0} u^{k} p_{k}, \quad \lambda p_{k}=\Pi(\{k\}), \quad \sum_{k \neq 0} p_{k}=1,
\end{gathered}
$$

respectively, where $\lambda$ and $p(u)=\mathrm{E} u^{\xi_{k}}$ are the intensity and moment generating function of the distribution function of jumps $\xi_{k}$ of the process $\xi(t)$. Denote by $\theta_{s}$ an exponential random variable such that $\mathrm{P}\left\{\theta_{s}>t\right\}=e^{-s t}$ for $s>0$ and $t>0$. Assume that $\theta_{s}$ is independent of $\xi(t)$. Then

$$
p(s, u)=\mathrm{E} u^{\xi\left(\theta_{s}\right)}=s \int_{0}^{\infty} e^{-s t} p_{t}(u) d t=\frac{s}{s-k(u)} .
$$

The lemma on the infinite divisibility holds for the moment generating function $p(s, u)$ (see [5] for the case of $n=1$, where $n$ is the number of states of the chain where the Poisson process $\xi(t)$ is defined).

2000 Mathematics Subject Classification. Primary 60G50, 60J70. 
Lemma 1. The following factorization holds for the moment generating function $p(s, u)$ :

$$
p(s, u)=p_{+}(s, u) p_{-}(s, u), \quad|u|=1,
$$

where

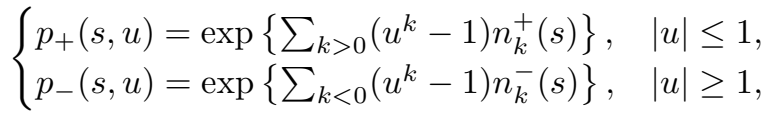

$$
\begin{aligned}
& n_{k}^{ \pm}(s)=\int_{0}^{\infty} t^{-1} e^{-s t} \rho_{ \pm k}(t) d t, \quad k \geq 0 \\
& \rho_{k}(t)=\mathrm{P}\{\xi(t)=k\}, \quad k=0, \pm 1, \ldots, \quad P_{t}(u)=\sum_{k=-\infty}^{\infty} u^{k} \rho_{k}(t)=\mathrm{E} u^{\xi(t)} .
\end{aligned}
$$

The distribution of the extrema of the process,

$$
\xi^{+}(t)=\sup _{0 \leq u \leq t} \xi(u) \quad \text { and } \quad \xi^{-}(t)=\inf _{0 \leq u \leq t} \xi(u),
$$

can be expressed in terms of the components of factorization (1) as follows:

$$
p_{ \pm}(s, u)=\mathrm{E} u^{\xi^{ \pm}\left(\theta_{s}\right)}=s \int_{0}^{\infty} \mathrm{E} u^{\xi^{ \pm}(t)} e^{-s t} d t .
$$

If $m_{1}=\mathrm{E} \xi(1)=\lambda \mathrm{E} \xi_{1}<0$, then

$$
\sum_{k>0} n_{k}^{+}(0)<\infty, \quad n_{k}^{+}(0)=\int_{0}^{\infty} t^{-1} \rho_{k}(t) d t<\infty
$$

and the global maximum

$$
\xi^{+}=\sup _{0 \leq t<\infty} \xi(t)=\lim _{t \rightarrow \infty} \xi^{+}(t)
$$

has a nondegenerate distribution with the moment generating function

$$
\begin{gathered}
p_{+}(u)=\mathrm{E} u^{\xi^{+}}=\exp \left\{\sum_{k>0}\left(u^{k}-1\right) n_{k}^{+}(0)\right\}, \\
p_{+}=\mathrm{P}\left\{\xi^{+}=0\right\}=p_{+}(0)=\exp \left\{-\sum_{k>0} n_{k}^{+}(0)\right\}>0 .
\end{gathered}
$$

If $m_{1}>0, n_{k}^{-}(0)<\infty$, and $\sum_{k<0} n_{k}^{-}(0)<\infty$, then the global minimum

$$
\xi^{-}=\inf _{0 \leq t<\infty} \xi(t)=\lim _{t \rightarrow \infty} \xi^{-}(t)
$$

has a nondegenerate distribution with the moment generating function

$$
\begin{aligned}
& p_{-}(u)=\mathrm{E} u^{\xi^{-}}=\exp \left\{\sum_{k<0}\left(u^{k}-1\right) n_{k}^{-}(0)\right\}, \\
& p_{-}=\mathrm{P}\left\{\xi^{-}=0\right\}=\exp \left\{-\sum_{k<0} n_{k}^{-}(0)\right\}>0 .
\end{aligned}
$$

Relation (3) is called the first factorization identity.

In contrast to the case of processes with a continuous phase space, there are two different versions of exit time moments in the case of processes with a discrete phase space. Let

$$
\tau^{+}(x)=\inf \{t: \xi(t)>x\}, \quad \bar{\tau}^{+}(x)=\inf \{t: \xi(t) \geq x\}, \quad x \geq 0 .
$$

The random variables $\tau^{+}(x)$ and $\bar{\tau}^{+}(x)$ are the first times when the process exits the sets $(-\infty, x]$ and $(-\infty, x)$, respectively, $x=0,1,2, \ldots$. Thus there are two different versions 
of the following functionals: the excess over a level at the exit time $\left(\gamma^{+}(x)\right.$ and $\left.\bar{\gamma}^{+}(x)\right)$, the undershoot of $x$ just before the exit time $\left(\gamma_{+}(x)\right.$ and $\left.\bar{\gamma}_{+}(x)\right)$, and the height of the first jump over a level $\left(\gamma_{x}^{+}\right.$and $\left.\bar{\gamma}_{x}^{+}\right)$, where

$$
\begin{cases}\gamma^{+}(x)=\xi^{+}\left(\tau^{+}(x)\right)-x, & \bar{\gamma}^{+}(x)=\xi^{+}\left(\bar{\tau}^{+}(x)\right)-x, \\ \gamma_{+}(x)=x-\xi^{+}\left(\tau^{+}(x)-0\right), & \bar{\gamma}_{+}(x)=x-\xi^{+}\left(\bar{\tau}^{+}(x)-0\right), \\ \gamma_{x}^{+}=\gamma^{+}(x)+\gamma_{+}(x), & \bar{\gamma}_{x}^{+}=\bar{\gamma}^{+}(x)+\bar{\gamma}_{+}(x), x \geq 0 .\end{cases}
$$

Let $\varepsilon_{\nu}$ be a geometrical random variable such that

$$
\mathrm{P}\left\{\varepsilon_{\nu}=k\right\}=(1-\nu) \nu^{k}, \quad k \geq 0,0<\nu<1 .
$$

Assume that $\varepsilon_{\nu}$ is independent of $\xi(t)$ and $\theta_{s}$. Then the following equality, also known as the second factorization identity, holds for $\left\{\tau^{+}(x), \gamma^{+}(x)\right\}$ and $\left\{\bar{\tau}^{+}(x), \bar{\gamma}^{+}(x)\right\}$ (see [4]).

Theorem 1. The joint distribution of $\left\{\tau^{+}(x), \gamma^{+}(x)\right\}$ and $\left\{\bar{\tau}^{+}(x), \bar{\gamma}^{+}(x)\right\}$ is given by

$$
\begin{aligned}
& \mathrm{E}\left[e^{-s \tau^{+}\left(\varepsilon_{\nu}\right)} u^{\gamma^{+}\left(\varepsilon_{\nu}\right)}, \tau^{+}\left(\varepsilon_{\mu}\right)<\infty\right]=\frac{(1-\nu) u}{u-\nu} \frac{\mathrm{E} u^{\xi^{+}\left(\theta_{s}\right)}-\mathrm{E} \nu^{\xi^{+}\left(\theta_{s}\right)}}{\mathrm{E} u u^{\xi^{+}\left(\theta_{s}\right)}}, \\
& \mathrm{E}\left[e^{-s \bar{\tau}^{+}\left(\varepsilon_{\nu}\right)} u^{\bar{\gamma}^{+}\left(\varepsilon_{\nu}\right)}, \bar{\tau}^{+}\left(\varepsilon_{\mu}\right)<\infty\right]=\frac{1-\nu}{u-\nu} \frac{u \mathrm{E} u^{\xi^{+}\left(\theta_{s}\right)}-\nu \mathrm{E} \nu^{\xi^{+}\left(\theta_{s}\right)}}{\mathrm{E} u^{\xi^{+}\left(\theta_{s}\right)}} .
\end{aligned}
$$

The proof of Theorem 1 is based on the following stochastic relations:

$$
\begin{aligned}
& \tau^{+}(n+z) \doteq \begin{cases}\tau^{+}(z), & \gamma^{+}(z)>n, \\
\tau^{+}(z)+\tau^{+}\left(n-\gamma^{+}(z)\right), & \gamma^{+}(z) \leq n,\end{cases} \\
& \bar{\tau}^{+}(n+z) \doteq \begin{cases}\bar{\tau}^{+}(z), & \bar{\gamma}^{+}(z)>n, \\
\bar{\tau}^{+}(z)+\bar{\tau}^{+}\left(n-\bar{\gamma}^{+}(z)\right), & \bar{\gamma}^{+}(z)<n .\end{cases}
\end{aligned}
$$

Denote by $\zeta_{1}$ and $\xi_{1}$ the time and height, respectively, of the first jump of the process $\xi(t)$; the joint moment generating function of $\left\{\tau^{+}(x), \gamma^{+}(x), \gamma_{+}(x), \gamma_{x}^{+}\right\}$and its moment generating transforms are denoted by

$$
\begin{gathered}
V(s, x, u, v, \mu)=\mathrm{E}\left[e^{-s \tau^{+}(x)} u^{\gamma^{+}(x)} v^{\gamma_{+}(x)} \mu^{\gamma_{x}^{+}}, \tau^{+}(x)<\infty\right], \\
v_{+}(s, \beta, u, v, \mu)=\sum_{x \geq 0} \beta^{x} V_{+}(s, x, u, v, \mu), \\
V_{+}(s, x, u, v, \mu)=V(s, x, u, v, \mu) \delta(x \geq 0),
\end{gathered}
$$

respectively. It is clear that

$$
\begin{aligned}
& \tau^{+}(x) \doteq\left\{\begin{array} { l l } 
{ \zeta _ { 1 } , } & { \xi _ { 1 } > x , } \\
{ \zeta _ { 1 } + \tau ^ { + } ( x - \zeta _ { 1 } ) , } & { \xi _ { 1 } \leq x , }
\end{array} \quad \overline { \tau } ^ { + } ( x ) \doteq \left\{\begin{array}{ll}
\zeta_{1}, & \xi_{1} \geq x, \\
\zeta_{1}+\bar{\tau}^{+}\left(x-\zeta_{1}\right), & \xi_{1}<x,
\end{array}\right.\right. \\
& \gamma^{+}(x) \doteq\left\{\begin{array} { l l } 
{ \xi _ { 1 } - x , } & { \xi _ { 1 } > x , } \\
{ \gamma ^ { + } ( x - \xi _ { 1 } ) , } & { \xi _ { 1 } \leq x , }
\end{array} \quad \overline { \gamma } ^ { + } ( x ) \doteq \left\{\begin{array}{ll}
\xi_{1}-x, & \xi_{1} \geq x \\
\bar{\gamma}^{+}\left(x-\xi_{1}\right), & \xi_{1}<x
\end{array}\right.\right. \\
& \gamma_{+}(x) \doteq\left\{\begin{array} { l l } 
{ x , } & { \xi _ { 1 } > x , } \\
{ \gamma _ { + } ( x - \xi _ { 1 } ) , } & { \xi _ { 1 } \leq x , }
\end{array} \quad \overline { \gamma } _ { + } ( x ) \doteq \left\{\begin{array}{ll}
x, & \xi_{1} \geq x \\
\bar{\gamma}_{+}\left(x-\xi_{1}\right), & \xi_{1}<x
\end{array}\right.\right. \\
& \gamma_{x}^{+} \doteq\left\{\begin{array} { l l } 
{ \xi _ { 1 } , } & { \xi _ { 1 } > x , } \\
{ \gamma _ { x - \xi _ { 1 } } ^ { + } , } & { \xi _ { 1 } \leq x , }
\end{array} \quad \overline { \gamma } _ { x } ^ { + } \doteq \left\{\begin{array}{ll}
\xi_{1}, & \xi_{1} \geq x \\
\bar{\gamma}_{x-\xi_{1}}^{+}, & \xi_{1}<x .
\end{array}\right.\right.
\end{aligned}
$$


Using these equalities we derive the following equation for $V_{+}(s, x)=V_{+}(s, x, u, v, \mu)$ :

$$
\begin{gathered}
(s+\lambda) V_{+}(s, x)-\lambda \sum_{k \neq 0} p_{k} V_{+}(s, x-k)=A_{x}(u, v, \mu), \quad x \geq 0, \\
A_{x}(u, v, \mu)=\lambda \sum_{k \geq x+1} u^{k-x} v^{x} \mu^{k} p_{k}, \quad x \geq 0,
\end{gathered}
$$

whence by taking the moment generating transforms in $x \geq 0$ we obtain the equation for $v_{+}(s, \beta)=v_{+}(s, \beta, u, v, \mu)$ :

$$
v_{+}(s, \beta)(s-k(\beta))=a(\beta, u, v, \mu)+\lambda\left[p(\beta) v_{+}(s, \beta)\right]_{-},
$$

where $[\cdot]_{ \pm}$and $[\cdot]_{ \pm}^{0}$ are the projectors defined by

$$
\begin{gathered}
{\left[\sum_{k=-\infty}^{\infty} \beta^{k} g_{k}\right]_{ \pm}=\sum_{ \pm k>0} g_{k} v^{k}, \quad \sum_{k=-\infty}^{\infty}\left|g_{k}\right|<\infty,} \\
{\left[\sum_{k=-\infty}^{\infty} g_{k} \beta^{k}\right]_{+}^{0}=\sum_{k \geq 0} g_{k} \beta^{k}, \quad a(\beta, u, v, \mu)=\sum_{x \geq 0} \beta^{x} A_{x}(u, v, \mu) .}
\end{gathered}
$$

Put

$$
\begin{gathered}
p_{k}^{ \pm}(s)=\mathrm{P}\left\{\xi^{ \pm}\left(\theta_{s}\right)=k\right\}, \quad \pm k \geq 0, p_{0}^{ \pm}(s)=p_{ \pm}(s), \\
G(s, x, u, v, \mu)=\sum_{k \leq 0} A_{x-k}(u, v, \mu) p_{k}^{-}(s), \quad x \geq 0 .
\end{gathered}
$$

The following result follows from Lemma 1 and equality (14).

Theorem 2. The moment generating transform of the moment generating function of the joint distribution of exit time functionals is given by the equality

$$
s v_{+}(\beta, s, u, v, \mu)=\left[p_{-}(s, \beta) a(\beta, u, v, \mu)\right]_{+}^{0} p_{+}(s, \beta),
$$

whence it follows that

$$
s V_{+}(s, x, u, v, \mu)=\sum_{r=0}^{x} G(s, x-r, u, v, \mu) p_{r}^{+}(s), \quad x \geq 0 .
$$

Proof. According to (3), equality (14) is equivalent to

$$
s v_{+}(s, \beta) p_{+}^{-1}(s, \beta)=\left\{a(\beta, u, v, \mu)+\lambda\left[p(\beta) v_{+}(s, \beta)\right]_{-}\right\} p_{-}(s, \beta) .
$$

Applying the projection operation $[\cdot]_{-}^{0}$ to the latter equality we get (15). Inverting (15) with respect to $\beta$ we prove (16).

An integer-valued compound Poisson process $\xi(t)$ is called lower continuous if the cumulant $k(u)$ in representation (1) for its moment generating function is given in terms of the moment generating function of the jump as follows

$$
p(u)=\sum_{k=-1}^{\infty} p_{k} u^{k}, \quad p_{-1}>0, p_{0}=0 .
$$

Similarly, a process $\xi(t)$ is called upper continuous if the moment generating function of its jumps is given by

$$
p(u)=\sum_{k \leq 1} p_{k} u^{k}, \quad p_{1}>0, p_{0}=0 .
$$

Factorization (3) can be written in an explicit form for lower continuous processes (see [6]). 
Lemma 2. The components of decomposition (3) for a lower continuous process $\xi(t)$ are given by

$$
\begin{gathered}
p_{-}(s, u)=\mathrm{E} u^{\xi^{-}\left(\theta_{s}\right)}=\frac{p_{-}(s)}{1-q_{-}(s) u^{-1}}, \quad q_{-}(s)=1-p_{-}(s), \quad|u| \geq 1, \\
p_{+}(s, u)=\mathrm{E} u^{\xi^{+}\left(\theta_{s}\right)}=\mathrm{P}\left\{\xi\left(\theta_{s}\right) \leq 0\right\} p_{0}^{-1}(s) \mathrm{E}\left[u^{\xi\left(\theta_{s}\right)}, \xi\left(\theta_{s}\right) \geq 0\right] \\
-\mathrm{P}\left\{\xi\left(\theta_{s}\right)<0\right\} p_{0}^{-1}(s) \mathrm{E}\left[u^{\xi\left(\theta_{s}\right)}, \xi\left(\theta_{s}\right)>0\right] \\
p_{k}(s)=\mathrm{P}\left\{\xi\left(\theta_{s}\right)=k\right\}, \quad k=0, \pm 1, \ldots, \quad p_{ \pm}(s)=\mathrm{P}\left\{\xi^{ \pm}\left(\theta_{s}\right)=0\right\}, \\
p_{m}^{+}(s)=\mathrm{P}\left\{\xi^{+}\left(\theta_{s}\right)=m\right\}=p_{-}^{-1}(s)\left[p_{m}(s)-p_{m+1}(s)\right]-p_{m+1}(s), \quad m \geq 0 .
\end{gathered}
$$

If $m_{1}<0$, then $p_{-}(s) \underset{s \rightarrow 0}{\longrightarrow} 0, s^{-1} p_{-}(s) \underset{s \rightarrow 0}{\longrightarrow} p_{-}^{\prime}(0)>0$. Moreover, the distribution of the global maximum is nondegenerate and is given by

$$
\begin{aligned}
& \left\{\begin{array}{l}
\mathrm{E} u^{\xi^{+}}=\frac{1}{p_{-}^{\prime}(0)} \int_{0}^{\infty}\left[\mathrm{E}\left(u^{\xi(t)}, \xi(t) \geq 0\right)-\mathrm{E}\left(u^{\xi(t)-1}, \xi(t) \geq 1\right)\right] d t, \\
\mathrm{P}\left\{\xi^{+}=k\right\}=\frac{1}{p_{-}^{\prime}(0)}\left(p_{k}^{\prime}(0)-p_{k+1}^{\prime}(0)\right), \quad k \geq 0,
\end{array}\right. \\
& p_{k}(s)=s \int_{0}^{\infty} e^{-s t} \mathrm{P}\{\xi(t)=k\} d t, \quad p_{k}^{\prime}(0)=\int_{0}^{\infty} \mathrm{P}\{\xi(t)=k\} d t .
\end{aligned}
$$

Applying (17), we find by inverting that

$$
p_{k}^{-}(s)=p_{-}(s) q_{-}^{-k}(s), \quad k \leq 0 .
$$

This result makes the evaluation of $G(s, x, u, v, \mu)$ easier (see (16)). In particular,

$$
\begin{gathered}
G_{1}(s, x, u)=G(s, x, u, 1,1)=\sum_{j \leq 0} A_{x-j}(u ; 1 ; 1) p_{j}^{-}(s) \\
=\frac{\lambda p_{-}(s)}{u-q_{-}(s)} \sum_{k \geq x+1} u p_{k}\left(u^{k-x}-q_{-}^{k-x}(s)\right), \quad x \geq 0, \\
G_{2}(s, x, v)=\sum_{j \leq 0} A_{x-j}(1, v, 1) p_{j}^{-}(s)=\lambda p_{-}(s) \sum_{k \geq x+1} q_{-}^{k-x-1}(s) v^{k-1} Q_{k}, \\
Q_{k}=\sum_{r \geq k} p_{r}, \quad k \geq 1, \\
G_{3}(s, x, \mu)=\sum_{j \leq 0} A_{x-j}(1,1, \mu) p_{j}^{-}(s)=\lambda \sum_{k \geq x+1} p_{k} \mu^{k}\left(1-q_{-}^{k-x}(s)\right), \quad x \geq 0 .
\end{gathered}
$$

Therefore, the moment generating functions with respect to $x$ can be written as follows:

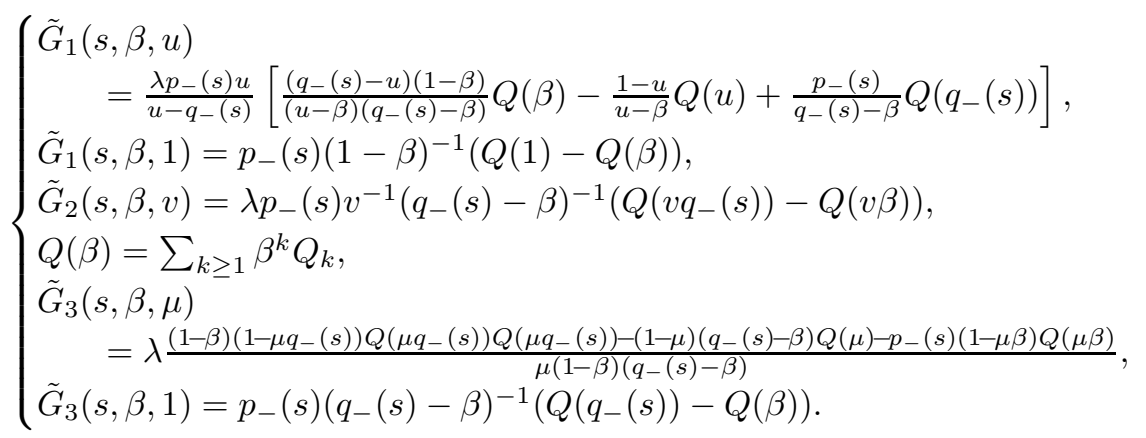

When evaluating the moment generating transforms we use the following notation and relations:

$$
E_{+} u^{\xi}=\mathrm{E}\left[u^{\xi}, \xi>0\right], \quad E_{+}\left(1-u^{\xi}\right)=(1-u) u^{-1} Q(u) .
$$


D. V. GUSAK

The function $G(s, x, u, v, \mu)$ and its moment generating function have a more complicated form, namely

$$
\left\{\begin{aligned}
& G(s, x, u, v, \mu)=\frac{\lambda u p_{-}(s)}{u-v q_{-}(s)} \sum_{k \geq x+1} p_{k} \mu^{k}\left[u^{k-x}(\beta v)^{x}-q^{k-x}(s)(\beta v)^{k}\right], \\
& \tilde{G}(s, \beta, u, v, \mu) \\
&=\frac{\lambda u p_{-}(s)}{u-v q_{-}(s)}\left\{\frac{u}{u-v \beta}\left[\frac{1-\beta v \mu}{\beta v \mu} Q(\beta v \mu)-\frac{1-\mu u}{(\mu u)} Q(\mu u)\right]\right. \\
&\left.\quad+\frac{q_{-}(s)}{q_{-}(s)-\beta v}\left[\frac{1-\mu q_{-}(s)}{\mu q_{-}(s)} Q\left(\mu q_{-}(s)\right)-\frac{1-\beta v \mu}{\beta v \mu} Q(\beta, v, \mu)\right]\right\} .
\end{aligned}\right.
$$

Denote by $\varepsilon_{\beta}$ a geometrical random variable with parameter $\beta, 0<\beta<1$. Assume that $\varepsilon_{\beta}$ is independent of $\xi(t)$ and $\theta_{s}$. It follows from $\mathrm{P}\left\{\tau^{+}(x)<t\right\}=\mathrm{P}\left\{\xi^{+}(t)>x\right\}$ that

$$
\begin{gathered}
\mathrm{E}\left[e^{-s \tau^{+}(x)}, \tau^{+}(x)<\infty\right]=\mathrm{P}\left\{\xi^{+}\left(\theta_{s}\right)>x\right\} \\
\mathrm{E}\left[e^{-s \tau^{+}\left(\varepsilon_{\beta}\right)}, \tau^{+}\left(\varepsilon_{\beta}\right)<\infty\right]=\mathrm{P}\left\{\xi^{+}\left(\theta_{s}\right)>\varepsilon_{\beta}\right\}=1-\mathrm{E} \beta^{\xi^{+}\left(\theta_{s}\right)} .
\end{gathered}
$$

Similar relations hold for $\bar{\tau}^{+}(x)$ :

$$
\begin{gathered}
\mathrm{P}\left\{\xi^{+}\left(\theta_{s}\right) \geq x\right\}=\mathrm{E}\left[e^{-s \bar{\tau}^{+}(x)}, \bar{\tau}^{+}(x)<\infty\right], \\
\mathrm{P}\left\{\xi^{+}\left(\theta_{s}\right) \geq \varepsilon_{\beta}\right\}=\mathrm{E}\left[e^{-s \bar{\tau}^{+}\left(\varepsilon_{\beta}\right)}, \bar{\tau}^{+}\left(\varepsilon_{\beta}\right)<\infty\right]=1-\beta \mathrm{E} \beta^{\xi^{+}\left(\theta_{s}\right)} .
\end{gathered}
$$

It follows from (23) that

$$
\begin{aligned}
\tilde{V}(s, \beta, u, v, \mu) & =\mathrm{E}\left[e^{-s \tau^{+}\left(s_{\beta}\right)} u^{\gamma^{+}\left(\varepsilon_{\beta}\right)} v^{\gamma_{+}\left(\varepsilon_{\beta}\right)} \mu^{\gamma_{\varepsilon_{\beta}}^{+}}, \tau^{+}\left(\varepsilon_{\beta}\right)<\infty\right] \\
& =\mathrm{E}\left[u^{\gamma^{+}\left(\varepsilon_{\beta}\right)} v^{\gamma_{+}\left(\varepsilon_{\beta}\right)} \mu^{\gamma_{\varepsilon_{\beta}}^{+}}, \xi^{+}\left(\theta_{s}\right)>\varepsilon_{\beta}\right]
\end{aligned}
$$

Put $\gamma_{1}(x)=\gamma^{+}(x), \gamma_{2}(x)=\gamma_{+}(x), \gamma_{3}(x)=\gamma_{x}^{+}$, and $u_{1}=u, u_{2}=v$, and $u_{3}=\mu$, respectively, for $\left|u_{k}\right| \leq 1, k=1,2,3$. Then

$$
\begin{aligned}
\tilde{V}_{k}\left(s, \beta, u_{k}\right) & =\mathrm{E}\left[e^{-s \tau^{+}\left(s_{\beta}\right)} u^{\gamma^{+}\left(\varepsilon_{\beta}\right)}, \tau^{+}\left(\varepsilon_{\beta}\right)<\infty\right]=\mathrm{E}\left[u_{k}^{\gamma_{k}\left(\varepsilon_{\beta}\right)} / \xi^{+}\left(\theta_{s}\right)>\varepsilon_{\beta}\right] \\
& =\frac{\tilde{V}_{k}\left(s, \beta, u_{k}\right)}{\mathrm{P}\left\{\xi^{+}\left(\theta_{s}\right)>\varepsilon_{\beta}\right\}}=\frac{\tilde{V}_{k}\left(s, \beta, u_{k}\right)}{1-\mathrm{E} \beta^{+}\left(\theta_{s}\right)} .
\end{aligned}
$$

Corollary 1. If a process $\xi(t)$ is lower continuous, then the moment generating function of the joint distribution of $\left\{\tau^{+}\left(\varepsilon_{\beta}\right), \gamma^{+}\left(\varepsilon_{\beta}\right), \gamma_{+}\left(\varepsilon_{\beta}\right), \gamma_{\varepsilon_{\beta}}^{+}\right\}$is given by

$$
s \tilde{V}(s, \beta, u, v, \mu)=\beta p_{+}(s, \beta) \tilde{G}(s, \beta, u, v) .
$$

Moreover

$$
\left\{\begin{array}{l}
s \tilde{V}_{k}\left(s, \beta, u_{k}\right)=\beta p_{+}(s, \beta) \tilde{G}\left(s, \beta, u_{k}\right), \quad k=1,2,3, \\
\mathrm{E}\left[u_{k}^{\gamma_{k}\left(\varepsilon_{\beta}\right)} / \xi^{+}\left(\theta_{s}\right)>\varepsilon_{\beta}\right]=\frac{s^{-1} \beta p_{+}(s, \beta) \tilde{G}_{k}\left(s, \beta, u_{k}\right)}{1-\mathrm{E} \beta^{\xi^{+}\left(\theta_{s}\right)}} .
\end{array}\right.
$$

The proof of Corollary 1 follows from (16) by applying relations (21)-(25) and taking the moment generating transforms with respect to $x$.

Corollary 2. Consider a lower continuous process. Then the distribution of $\xi^{+}\left(\theta_{s}\right)$ is determined by its moment generating function:

$$
\left\{\begin{array}{l}
p_{+}(s, \beta)=1\left(1+\frac{1-\beta}{s} \tilde{G}_{1}(s, \beta, 1)\right)^{-1} \\
p_{+}(s)=p_{+}(s, 0)=\left[1+\frac{\lambda p_{-}(s)}{s q_{-}(s)} Q\left(q_{-}(s)\right)\right]^{-1}>0
\end{array}\right.
$$




$$
\tilde{G}_{1}(s, \beta, 1)=\frac{\lambda p_{-}(s)}{q_{-}(s)-\beta}\left(Q\left(q_{-}(s)\right)-Q(\beta)\right) .
$$

The distribution of the global maximum $\xi^{+}$is nondegenerate if $m_{1}=\lambda\left(Q(1)-p_{-1}\right)<0$. In this case,

$$
\left\{\begin{array}{l}
\mathrm{E} \beta^{\xi^{+}}=\lim _{s \rightarrow 0} p_{+}(s, \beta)=\left[1-\frac{\lambda}{\left|m_{1}\right|} \sum_{k=1}^{\infty}\left(\beta^{k}-1\right) Q_{k}\right]^{-1}, \\
p_{+}=\mathrm{P}\left\{\xi^{+}=0\right\}=\left(1+\frac{\lambda}{\left|m_{1}\right|} \sum_{k=1}^{\infty} Q_{k}\right)^{-1}=\left(\lambda p_{-1}\right)^{-1} .
\end{array}\right.
$$

The proof of (28) is based on the first relation in (27) for $u_{k}=1$. Passing to the limit as $s \rightarrow 0$ we derive (29) from (28) if $m_{1}<0$. Relation (29) can be written in terms of the cumulant of a nondecreasing integer-valued process $\xi_{*}(t)$ as follows:

$$
\psi_{*}(\beta)=\frac{\lambda}{\left|m_{1}\right|} \sum\left(\beta^{k}-1\right) Q_{k}, \quad \mathrm{E} \beta^{\xi_{*}(t)}=e^{t \psi_{*}(\beta)} .
$$

If $P\left\{\theta_{1}>t\right\}=\exp \{-t\}$, then

$$
\mathrm{E} \beta^{\xi^{+}}=\left(1-\psi_{*}(\beta)\right)^{-1}=\mathrm{E} \beta^{\xi_{*}\left(\theta_{1}\right)}=\int_{0}^{\infty} e^{-t} e^{t \psi_{*}(\beta)} d t
$$

Equality (30) implies a result of the Pollaczek-Spitzer type:

$$
\mathrm{E} \beta^{\xi^{+}}=\frac{p_{+}}{1-q_{+} p_{*}(\beta)}, \quad p_{*}(\beta)=\frac{Q(\beta)}{Q(1)}, \quad p_{+}=\frac{1}{\lambda p_{-1}}, \quad q_{+}=1-p_{+} .
$$

Comparing (5) or (19) with (28), it is obvious that relation (28) is more convenient for evaluating the distribution of $\xi^{+}\left(\theta_{s}\right)$. Similarly, relations (29)-(31) are more convenient for evaluating the distribution of $\xi^{+}$than relations (6) or (20) in the case of $m_{1}<0$. Equating probabilities $p_{+}$from (20) and (29) for $k=0$ we prove that

$$
p_{-}^{\prime}(0)=\lambda p_{-1}\left[p_{0}^{\prime}(0)-p_{1}^{\prime}(0)\right] .
$$

Now we consider the distributions of exit time functionals of $\left\{\tau^{+}(0), \gamma_{k}^{+}(0), k=1,2,3\right\}$ and limit distributions of $\left\{\tau^{+}(x), \gamma_{k}^{+}(x)\right\}$ as $x \rightarrow \infty$.

Corollary 3. The joint moment generating function of $\left\{\tau^{+}(0), \gamma_{k}(0)\right\}, k=1,2,3$,

$$
\mathrm{E}\left[e^{-s \tau^{+}(0)} u^{\gamma_{k}(0)}, \tau^{+}(0)<\infty\right]=\mathrm{E}\left[u^{\gamma_{k}(0)}, \xi^{+}\left(\theta_{s}\right)>0\right]
$$

is given by

$$
\begin{gathered}
\mathrm{E}\left[u^{\gamma^{+}(0)}, \xi^{+}\left(\theta_{s}\right)>0\right] \\
=\frac{\lambda p_{+}(s) p_{-}(s)}{s\left(u-q_{-}(s)\right) q_{-}(s)}\left[u p_{-}(s) Q\left(q_{-}(s)\right)-(1-u) q_{-}(s) Q(u)\right], \\
\mathrm{P}\left\{\gamma^{+}(0) \geq r, \xi^{+}\left(\theta_{s}\right)>0\right\}=\lambda p_{+}(s) p_{-}(s) s^{-1} \sum_{j=r}^{\infty} q_{-}^{j-r}(s) Q_{j}, \quad r \geq 1,
\end{gathered}
$$

if $\gamma_{1}(0)=\gamma^{+}(0)$, or by

$$
\begin{gathered}
\mathrm{E}\left[v^{\gamma_{+}(0)}, \xi^{+}\left(\theta_{s}\right)>0\right]=\lambda p_{+}(s) p_{-}(s) s^{-1} \sum_{k=1}^{\infty} q_{-}^{k-1}(s) v^{k-1} Q_{k}, \\
\mathrm{P}\left\{\gamma_{+}(0)=k, \xi^{+}\left(\theta_{s}\right)>0\right\}=\lambda p_{+}(s) p_{-}(s) s^{-1} q_{-}^{k+1} Q_{k+1}, \quad k \geq 0,
\end{gathered}
$$


if $\gamma_{2}(0)=\gamma_{+}(0)$, or by

$$
\begin{gathered}
\mathrm{E}\left[\mu^{\gamma_{0}^{+}}, \xi^{+}\left(\theta_{s}\right)>0\right]=\lambda p_{+}(s) s^{-1} \sum_{k=1}^{\infty} p_{k} \mu^{k}\left(1-q_{-}^{k}(s)\right), \\
\mathrm{P}\left\{\gamma_{0}^{+}=k, \xi^{+}\left(\theta_{s}\right)>0\right\}=\lambda p_{+}(s) s^{-1} p_{k}\left(1-q_{-}^{k}(s)\right), \quad k \geq 1,
\end{gathered}
$$

if $\gamma_{3}(0)=\gamma_{0}^{+}$.

Proof. According to (16), the moment generating functions

$$
V_{k}(s, x, u)=\mathrm{E}\left[e^{-s \tau^{+}(x)} u^{\gamma_{k}(x)}, \tau^{+}(x)<\infty\right]
$$

are given by

$$
s V_{k}(s, x, u)=\sum_{r=0}^{x} G_{k}(s, x-r, u) p_{r}^{+}(s), \quad x \geq 0 .
$$

We get that for $x=0$

$$
s V_{k}(s, 0, u)=p_{+}(s) G_{k}(s, 0, u), \quad k=1,2,3,
$$

where $G_{k}(s, 0, u), k=1,2,3$, are defined by (20). Relations (33), (35), and (37) follow from (40). Inverting (33), (35), and (37) we obtain distributions (34), (36), and (38), respectively.

It follows from (34) for $r=1$ (alternatively, from (35) for $v=1$ ) that

$$
s q_{+}(s) q_{-}(s)=\lambda p_{-}(s) p_{+}(s) Q\left(q_{-}(s)\right) .
$$

If $m_{1}>0$, then $p_{+}(s) \rightarrow 0, q_{+}(s) \rightarrow 1, p_{-}(s) \rightarrow p_{-}>0$, and $q_{-}(s) \rightarrow q_{-}>0$ as $s \rightarrow 0$. From the latter relation we find that

$$
\lim _{s \rightarrow 0} s^{-1} p_{+}(s)=p_{+}^{\prime}(0)=\left(\lambda p_{-} Q\left(q_{-}\right)\right)^{-1} .
$$

If $m_{1}=0$, then $p_{ \pm}(s) \rightarrow 0$ and $q_{ \pm}(s) \rightarrow 1$ as $s \rightarrow 0$. Since $m_{1}=\lambda\left(Q(1)-p_{-1}\right)=0$ $\left(p_{-1}=Q(1)\right)$, we find in the same way that $p_{ \pm}(s) \approx C_{ \pm} \sqrt{s}$ and

$$
\lim _{s \rightarrow 0} s^{-1} p_{+}(s) p_{-}(s)=(\lambda Q(1))^{-1}=\left(\lambda p_{-1}\right)^{-1} .
$$

Note that

$$
\mathrm{P}\left\{\tau^{+}(x)<\infty\right\}=1
$$

for $m_{1} \geq 0$, thus

$$
V_{k}\left(s, x, u_{k}\right)=\mathrm{E}\left[e^{-s \tau^{+}(x)} u_{k}^{\gamma_{k}(x)}\right], \quad k=1,2,3 .
$$

If $m_{1}<0$, then

$$
\mathrm{P}\left\{\tau^{+}(x)<\infty\right\}<1
$$

and

$$
\mathrm{P}\left\{\tau^{+}(x)<\infty\right\} \rightarrow 0
$$

as $x \rightarrow \infty$. We use relations (25) and (27) for conditional moment generating functions to study the limit distributions of $\left\{\tau^{+}(x), \gamma_{k}(x)\right\}$ as $x \rightarrow \infty$. These limit distributions can be found by passing to the limit as $s \rightarrow 0$ and $\beta \rightarrow 1$ for $m_{1} \geq 0$. In the case of $m_{1}<0$, the conditional limit distributions can be found by passing to the limit in relations (27) as $s \rightarrow 0$ and $\beta \rightarrow 1$. 
Corollary 4. Let $m_{1} \geq 0$. The joint distribution of $\left\{\tau^{+}\left(\varepsilon_{\beta}\right), \gamma_{k}\left(\varepsilon_{\beta}\right)\right\}$ is given by

$$
\mathrm{E}\left[e^{-s \tau^{+}\left(\varepsilon_{\beta}\right)} u^{\gamma_{k}\left(\varepsilon_{\beta}\right)}\right]=(1-\beta) s^{-1} p_{+}(s, \beta) \tilde{G}_{k}(s, \beta, u), \quad k=1,2,3,
$$

$\left(\tilde{G}_{k}(s, \beta, u)\right.$ is defined by (21)) and

$$
\mathrm{E} u^{\gamma_{k}\left(\varepsilon_{\beta}\right)}=\lim _{s \rightarrow 0} \frac{\tilde{G}_{k}(s, \beta, u)}{\tilde{G}_{k}(s, \beta, 1)}, \quad k=1,2,3 .
$$

Moreover if $m_{1}>0$, then

$$
p_{-}(s) \rightarrow p_{-}>0, \quad q_{-}(s) \rightarrow q, \quad\left(p_{-}(s)+q_{-}(s)=1\right),
$$

and thus the right-hand sides of (42) take the form

$$
\begin{aligned}
& \lim _{s \rightarrow 0} \frac{\tilde{G}_{1}(s, \beta, u)}{\tilde{G}_{1}(s, \beta, 1)}= \frac{u}{u-q_{-}}\left[\frac{\left(q_{-}-u\right)(1-\beta)}{(u-\beta)\left(q_{-}-\beta\right)} Q(\beta)-\frac{1-u}{u-\beta} Q(u)-\frac{p_{-} Q\left(q_{-}\right)}{q_{-}-\beta}\right] \\
& \times \frac{q_{-}-\beta}{Q\left(q_{-}\right)-Q(\beta)}, \\
& \lim _{s \rightarrow 0} \frac{\tilde{G}_{2}(s, \beta, u)}{\tilde{G}_{2}(s, \beta, 1)}=\frac{1}{v} \lim _{s \rightarrow 0} \frac{Q\left(v q_{-}(s)\right)-Q(\beta v)}{Q\left(q_{-}(s)\right)-Q(v)}=\frac{1}{v} \frac{Q\left(v q_{-}\right)-Q(\beta v)}{Q\left(q_{-}\right)-Q(\beta)}, \\
& \lim _{s \rightarrow 0} \frac{\tilde{G}_{3}(s, \beta, \mu)}{\tilde{G}_{3}(s, \beta, 1)} \\
&=\frac{(1-\beta)\left(1-\mu q_{-}\right) Q\left(\mu q_{-}\right)-(1-\mu)\left(q_{-}-\beta\right) Q(\mu)-p_{-}(1-\mu \beta) Q(\beta \mu)}{\mu p_{-}(1-\beta)\left(Q\left(q_{-}\right)-Q(\beta)\right)} .
\end{aligned}
$$

Approaching the limit in relations (42)-(45) as $\beta \rightarrow 1$, we determine the moment generating functions $\gamma_{k}(\infty)$ in the case of $m_{1}>0$ :

$$
\begin{gathered}
\mathrm{E} u^{\gamma^{+}(\infty)}=\frac{p_{-} u}{u-q_{-}}\left(Q(u)-Q\left(q_{-}\right)\right)\left(Q(1)-Q\left(q_{-}\right)\right)^{-1}, \\
\mathrm{E} v^{\gamma_{+}(\infty)}=\frac{1}{v}\left(Q\left(v q_{-}\right)-Q(v)\right)\left(Q\left(q_{-}\right)-Q(1)\right)^{-1}, \\
\mathrm{E} \mu^{\gamma_{\infty}^{+}}=\sum_{k=1}^{\infty} p_{k}\left(k-\sum_{r=1}^{k} q_{-}^{r}\right) \mu^{k}\left(Q(1)-Q\left(q_{-}\right)\right)^{-1} .
\end{gathered}
$$

The distributions of $\gamma_{k}(\infty), k=1,2,3$, are of the following form if $m_{1}>0$ :

$$
\begin{cases}\mathrm{P}\left\{\gamma^{+}(\infty)=n\right\}=\sum_{k=n}^{\infty} Q_{k} q_{-}^{k-n-1}\left(Q(1)-Q\left(q_{-}\right)\right)^{-1}, & n \geq 1, \\ \mathrm{P}\left\{\gamma_{+}(\infty)=n\right\}=Q_{n+1}\left(1-q_{-}^{n+1}\right)\left(Q(1)-Q\left(q_{-}\right)\right)^{-1}, & n \geq 0, \\ \mathrm{P}\left\{\gamma_{\infty}^{+}=n\right\}=p_{n}\left(n-\sum_{r=1}^{n} q_{-}^{r}\right)\left(Q(1)-Q\left(q_{-}\right)\right)^{-1}, & n \geq 1 .\end{cases}
$$

Proof. Relation (41) follows from (39) by applying moment generating transforms with respect to $x$. Then we have that for $m_{1} \geq 0$,

$$
\begin{aligned}
& \mathrm{E}\left[e^{-s \tau^{+}\left(\varepsilon_{\beta}\right)} u^{\gamma_{k}\left(\varepsilon_{\beta}\right)}, \tau^{+}\left(\varepsilon_{\beta}\right)<\infty\right] \\
& =\mathrm{E}\left[e^{-s \tau^{+}\left(\varepsilon_{\beta}\right)} u^{\gamma_{k}\left(\varepsilon_{\beta}\right)}\right]=\frac{(1-\beta) \tilde{G}_{k}(s, \beta, u)}{s+(1-\beta) \tilde{G}_{k}(s, \beta, 1)}, \quad k=1,2,3 .
\end{aligned}
$$

Now passing to the limit as $s \rightarrow 0$ we prove (42) for $m_{1} \geq 0$. The limits (43)-(45) can be evaluated for $\beta<1$ from (21) for $m_{1}>0$. Relations (43), (44), and (45) determine the moment generating functions of $\gamma_{1}\left(\varepsilon_{\beta}\right)=\gamma^{+}\left(\varepsilon_{\beta}\right), \gamma_{2}\left(\varepsilon_{\beta}\right)=\gamma_{+}\left(\varepsilon_{\beta}\right)$, and $\gamma_{3}\left(\varepsilon_{\beta}\right)=\gamma_{\varepsilon_{\beta}}^{+}$, respectively. Passing to the limit as $\beta \rightarrow 1$ in (43)-(45), we obtain the moment generating 
functions of $\gamma_{k}(\infty)(k=1,2,3)$. Relations (46)-(48) can be proved in the same way. Inverting (46)-(48), we determine the distributions of $\gamma_{k}(\infty)$ for $m_{1}>0$.

Since

$$
p_{ \pm}(s) \rightarrow 0 \quad \text { and } \quad q_{ \pm}(s) \rightarrow 1
$$

as $s \rightarrow 0$, we obtain the corresponding results for $m_{1}=0$.

Corollary 5. Let $m_{1}=0$. The moment generating functions of $\gamma_{k}\left(\varepsilon_{\beta}\right)$ are given by

$$
\begin{gathered}
\mathrm{E} u^{\gamma^{+}\left(\varepsilon_{\beta}\right)}=\lim _{s \rightarrow 0} \frac{\tilde{G}_{1}(s, \beta, u)}{\tilde{G}_{1}(s, \beta, 1)}=\frac{u(1-\beta)}{u-\beta} \frac{Q(u)-Q(\beta)}{Q(1)-Q(\beta)}, \\
\mathrm{E} v^{\gamma_{+}\left(\varepsilon_{\beta}\right)}=\lim _{s \rightarrow 0} \frac{\tilde{G}_{2}(s, \beta, u)}{\tilde{G}_{2}(s, \beta, 1)}=\frac{1}{v}(Q(v)-Q(\beta v))(Q(1)-Q(\beta))^{-1}, \\
\mathrm{E} \mu^{\gamma_{\varepsilon_{\beta}}^{+}}=\lim _{s \rightarrow 0} \frac{\tilde{G}_{3}(s, \beta, \mu)}{\tilde{G}_{3}(s, \beta, 1)} \\
=\frac{(1-\beta \mu) Q(\mu)-(1-\beta)(1-\mu) \mu Q^{\prime}(\mu)-(1-\mu \beta) Q(\mu \beta)}{\mu(1-\beta)(Q(1)-Q(\beta))} .
\end{gathered}
$$

The moment generating functions of $\gamma_{k}(\infty)$ are given by

$$
\left\{\begin{array}{l}
\mathrm{E} u^{\gamma^{+}(\infty)}=u(Q(1)-Q(u))\left[(1-u) Q^{\prime}(1)\right]^{-1}, \\
\mathrm{E} v^{\gamma_{+}(\infty)}=Q^{\prime}(v) Q^{\prime}(1)^{-1}, \quad \mathrm{P}\left\{\gamma_{+}(\infty)=0\right\}=p_{1} Q^{\prime}(1)^{-1}, \\
\mathrm{E} \mu^{\gamma_{\infty}^{+}}=\left[\mu Q^{\prime}(\mu)-\frac{1}{2} \mu(1-\mu) Q^{\prime \prime}(\mu)\right] Q^{\prime}(1)^{-1} .
\end{array}\right.
$$

The distributions of $\gamma_{k}(\infty), k=1,2,3$, are of the form

$$
\begin{cases}\mathrm{P}\left\{\gamma^{+}(\infty)=n\right\}=\bar{Q}_{n} Q^{\prime}(1)^{-1}, \quad \bar{Q}_{n}=\sum_{k=n}^{\infty} Q_{n}, & n \geq 1, \\ \mathrm{P}\left\{\gamma_{+}(\infty)=n\right\}=(n+1) p_{n+1} Q^{\prime}(1)^{-1}, & n \geq 0, \\ \mathrm{P}\left\{\gamma_{\infty}^{+}=n\right\}=n(n+1) p_{n} Q^{\prime}(1)^{-1}, & n \geq 1 .\end{cases}
$$

Proof. If $m_{1}=0$, then the moment generating functions of $\gamma_{k}\left(\varepsilon_{\beta}\right)$ can be determined from (42) by passing to the limit as $s \rightarrow 0$. Note that $q_{-}(s)=1-p_{-}(s) \rightarrow 1$ as $s \rightarrow 0$ and thus it is easy to evaluate $\lim _{s \rightarrow 0} \tilde{G}_{1}(s, \beta, u)$ as well as to find the distribution of $\gamma_{1}\left(\varepsilon_{\beta}\right)$. When evaluating the $\operatorname{limits}_{\lim _{s \rightarrow 0}} \tilde{G}_{2,3}(s, \beta, u) \tilde{G}_{2,3}^{-1}(s, \beta, 1)$ one must take into account that

$$
Q\left(u q_{-}(s)\right)=Q(u)-u p_{-}(s) Q^{\prime}(u)+o\left(p_{-}(s)\right), \quad s \rightarrow 0,
$$

whence relations (51)-(53) follow. Now we pass to the limit as $\beta \rightarrow 1$ to determine the moment generating functions of $\gamma_{1,2}(\infty)$ from (51)-(52) (see (54)). When passing to the limit in (53) as $\beta \rightarrow 1$ we use the expansion with respect to $\delta=1-\beta \rightarrow 0$, namely

$$
Q(u \beta)=Q(u)-u \delta Q^{\prime}(u)-\frac{1}{2} u^{2} \delta^{2} Q^{\prime \prime}(u)+o\left(\delta^{2}\right) .
$$

This allows us to obtain the last relation in (54). Indeed, inverting (54) we prove (55). When inverting the moment generating function of $\gamma_{\infty}^{+}$we use the equality

$$
p_{n}=Q_{n}-Q_{n+1} \text {. }
$$

After some transformations of a relation for $\gamma_{3}(\infty)=\gamma_{\infty}^{+}$we obtain the last relation in (54) from

$$
\mathrm{E} \mu^{\gamma_{\infty}^{+}}=\sum_{n=1}^{\infty} n(n+1)\left(Q_{n}-Q_{n+1}\right) \mu^{n} Q^{\prime}(1)^{-1}
$$


Corollary 6. Assume that $m_{1}<\infty$. Then the conditional moment generating functions of $\left\{\tau^{+}\left(\varepsilon_{\beta}\right), \gamma_{k}\left(\varepsilon_{\beta}\right)\right\}$ are given by

$$
\begin{gathered}
\mathrm{E}\left[u^{\gamma_{+}\left(\varepsilon_{\beta}\right)} e^{-s \tau^{+}\left(\varepsilon_{\beta}\right)} / \tau^{+}\left(\varepsilon_{\beta}\right)<\theta_{s}\right]=\frac{\beta}{1-\mathrm{E} \beta^{\xi^{+}\left(\theta_{s}\right)}} \frac{\tilde{G}_{k}(s, \beta, u)}{s+(1-\beta) \tilde{G}_{k}(s, \beta, 1)}, \\
\mathrm{E}\left[u^{\gamma_{+}(\infty)} e^{-s \tau^{+}(\infty)} / \tau^{+}(\infty)<\theta_{s}\right]=\tilde{G}_{k}(s, 1, u)\left(s \mathrm{E} \xi^{+}\left(\theta_{s}\right)\right)^{-1}, \quad k=1,2,3 .
\end{gathered}
$$

The limit conditional moment generating functions of $\gamma_{1}(\infty)=\gamma^{+}(\infty), \gamma_{2}(\infty)=\gamma_{+}(\infty)$, $\gamma_{3}(\infty)=\gamma_{\infty}^{+}$, and $\tau^{+}(\infty)$ are such that

$$
\left\{\begin{array}{l}
\mathrm{E}\left[u^{\gamma^{+}(\infty)} e^{-s \tau^{+}(\infty)} / \tau^{+}(\infty)<\theta_{s}\right]=\frac{\lambda p_{-}(s)}{s \mathrm{E} \xi^{+}\left(\theta_{s}\right)\left(u-q_{-}(s)\right)}\left[Q(u)-Q\left(q_{-}(s)\right)\right], \\
\mathrm{E}\left[v^{\gamma^{+}(\infty)} e^{-s \tau^{+}(\infty)} / \tau^{+}(\infty)<\theta_{s}\right]=\frac{\lambda p_{-}(s)}{s v \mathrm{E} \xi^{+}\left(\theta_{s}\right)\left(q_{-}(s)-1\right)}\left[Q\left(v q_{-}(s)\right)-Q(v)\right], \\
\mathrm{E}\left[\mu^{\gamma_{\infty}^{+}} e^{-s \tau^{+}(\infty)} / \tau^{+}(\infty)<\theta_{s}\right] \\
\quad=\frac{\lambda}{s \mathrm{E} \xi^{+}\left(\theta_{s}\right)} \\
\quad \times\left[\frac{1-\mu}{\mu p_{-}(s)}\left(Q(\mu)-Q\left(q_{-}(s) \mu\right)\right)+Q(\mu)-Q\left(q_{-}(s) \mu\right)-(1-\mu) Q^{\prime}(\mu)\right] .
\end{array}\right.
$$

The conditional moment generating functions of $\left\{\gamma_{k}(\infty), k=1,2,3\right\}$ are given by

$$
\left\{\begin{array}{l}
\mathrm{E}\left[u^{\gamma^{+}(\infty)} / \tau^{+}(\infty)<\infty\right]=\frac{\lambda p_{-}^{\prime}(0) u}{\mathrm{E} \xi^{+}(1-u)}(Q(1)-Q(u)), \\
\left.\mathrm{E}\left[v^{\gamma+(}\right) / \tau^{+}(\infty)<\infty\right]=\lambda p_{-}^{\prime}(0)\left(\mathrm{E} \xi^{+}\right)^{-1} Q^{\prime}(v), \quad \mathrm{E} \xi^{+}=\lambda p_{-}^{\prime}(0) Q^{\prime}(1), \\
\mathrm{E}\left[\mu^{\gamma_{\infty}^{+}} / \tau^{+}(\infty)<\infty\right]=\lambda p_{-}^{\prime}(0)\left(\mathrm{E} \xi^{+}\right)^{-1} \mu\left[Q^{\prime}(\mu)-\frac{1}{2}(1-\mu) Q^{\prime \prime}(\mu)\right] .
\end{array}\right.
$$

Proof. Using (27) and (28) we prove relations (56), whence (57) follows by passing to the limit as $\beta \rightarrow 1$. Applying (21) we find the functions

$$
\begin{gathered}
\tilde{G}_{1}(s, 1, u)=\frac{\lambda p_{-}(s)}{u-q_{-}(s)}\left[Q(u)-Q\left(q_{-}(s)\right)\right], \\
\tilde{G}_{2}(s, 1, v)=\frac{\lambda p_{-}(s)}{v\left(q_{-}(s)-1\right)}\left[Q\left(v q_{-}(s)\right)-Q(v)\right], \\
\tilde{G}_{3}(s, 1, \mu) \\
=\lambda\left[\frac{(1-\mu)\left(Q(\mu)-Q\left(q_{-}(s) \mu\right)\right)}{\mu} p_{-}(s)+Q(\mu)-Q\left(q_{-}(s) \mu\right)-(1-\mu) Q^{\prime}(\mu)\right] .
\end{gathered}
$$

These functions determine the right-hand sides of relations (58). Passing to the limit in (58) as $s \rightarrow 0$ and taking into account that $p_{-}(s) \rightarrow 0$ and

$$
p_{-}^{\prime}(0)=\lim _{s \rightarrow 0} s^{-1} p_{-}(s)>0
$$

we find the conditional moment generating functions $\mathrm{E}\left[u^{\gamma^{+}(\infty)} / \tau^{+}(\infty)<\infty\right]$ in (59):

$$
\begin{gathered}
\lim _{s \rightarrow 0} s^{-1} \tilde{G}_{1}(s, 1, u)=\frac{\lambda p_{-}^{\prime}(0) u}{1-u}(Q(1)-Q(u)), \\
\lim _{s \rightarrow 0} s^{-1} \tilde{G}_{2}(s, 1, v)=\lambda p_{-}^{\prime}(0) Q^{\prime}(v), \\
\lim _{s \rightarrow 0} s^{-1} \tilde{G}_{3}(s, 1, \mu)=\lambda p_{-}^{\prime}(0)\left[\mu Q^{\prime}(\mu)-\frac{1}{2} \mu(1-\mu) Q^{\prime \prime}(\mu)\right] .
\end{gathered}
$$

Relations (59) can be inverted in the same way as relations (54). Thus the conditional distributions of $\gamma_{k}(\infty)$,

$$
\mathrm{P}\left\{\gamma_{k}(\infty)=n / \tau^{+}(\infty)<\infty\right\}, \quad k=1,2,3,
$$

are determined for $m_{1}=\left(Q(1)-p_{-}\right) \lambda<0$ by the right-hand sides in $(55)$. 
Applying Theorem 1 one can derive for $m_{1} \geq 0$ a relation between $\gamma^{+}(0)$ and $\gamma^{+}(\infty)$. A similar result for processes with a continuous phase space is presented by Corollary 2.2 in [7].

Corollary 7. Let $m_{1} \geq 0$. Then

$$
\mathrm{E} u^{\gamma^{+}(\infty)}=\frac{u}{\mathrm{E} \gamma^{+}(0)(1-u)}\left(1-\mathrm{E} u^{\gamma^{+}(0)}\right) .
$$

Proof. Let $m_{1} \geq 0$. Using relation (10) and approaching the limit as $\nu \rightarrow 0$ we get

$$
\mathrm{E}\left[e^{-s \tau^{+}(0)} u^{\gamma^{+}(0)}\right]=1-\frac{p_{+}(s)}{\mathrm{E} u^{\xi^{+}\left(\theta_{s}\right)}} .
$$

This means that for $m_{1} \geq 0$,

$$
\mathrm{E} u^{\xi^{+}\left(\theta_{s}\right)} \approx \frac{p_{+}(s)}{1-\mathrm{E} u^{\gamma^{+}(0)}} \quad \text { as } \quad s \rightarrow 0 .
$$

Substituting the corresponding approximations for the moment generating functions of $\xi^{+}\left(\theta_{s}\right)$ in (10), we obtain

$$
\mathrm{E} u^{\gamma^{+}\left(\varepsilon_{\nu}\right)}=\frac{(1-\nu) u}{u-\nu}\left[1-\frac{1-\mathrm{E} u^{\gamma^{+}(0)}}{1-\mathrm{E} \nu^{\gamma^{+}(0)}}\right]=\frac{(1-\nu) u}{u-\nu} \frac{\mathrm{E} u^{\gamma^{+}(0)}-\mathrm{E} \nu^{\gamma^{+}(0)}}{1-\mathrm{E} \nu^{\gamma^{+}(0)}} .
$$

Passing to the limit as $\nu \rightarrow 1$ we get (60).

It is worth mentioning that the pair $\left\{\bar{\tau}^{+}(0), \bar{\gamma}^{+}(0)\right\}$ has degenerate distribution, namely

$$
\mathrm{P}\left\{\bar{\tau}^{+}(0)=\bar{\gamma}^{+}(0)=0\right\}=1 \text {. }
$$

The last result follows from the definition of the above functionals and agrees with a relation obtained from (11) as $\nu \rightarrow 0$.

We introduce the following notation:

$$
\begin{gathered}
\bar{V}(s, x, u, v, \mu)=\mathrm{E}\left[e^{-s \bar{\tau}^{+}(x)} u^{\bar{\gamma}^{+}(x)} v^{\bar{\gamma}_{+}(x)} \mu^{\bar{\gamma}_{x}^{+}}, \bar{\tau}^{+}(x)<\infty\right], \\
\bar{v}_{+}(s, \beta, u, v, \mu)=\sum_{x \geq 0} \beta^{x} \bar{V}(s, x, u, v, \mu) .
\end{gathered}
$$

Denote the functions that give the right-hand sides of equations similar to (13) but with $\bar{V}(s, x)$ instead of $V_{+}(s, x)$, by

$$
\begin{gathered}
\bar{A}_{x}(u, v, \mu)=\lambda \sum_{k=x}^{\infty} u^{k-x} v^{x} \mu^{k} p_{k}, \quad x \geq 0, \\
\bar{a}(\beta, u, v, \mu)=\sum_{x \geq 0} \beta^{x} \bar{A}_{x}(u, v, \mu), \\
\bar{G}(s, x, u, v, \mu)=\sum_{k \leq 0} \bar{A}_{k-x}(u, v, \mu) p_{k}^{-}(s), \quad x \geq 0 .
\end{gathered}
$$

Similarly to Theorem 2 one can prove

Theorem 3. The moment generating transform of the joint moment generating function of the functionals $\left\{\bar{\tau}^{+}(x), \bar{\gamma}^{+}(x), k=1,2,3\right\}$ is given by

$$
s \bar{V}_{+}(s, \beta, v, \mu)=\left[p_{-}(s, \beta) \bar{a}(\beta, u, v, \mu)\right]_{+}^{0} p_{+}(s, \beta),
$$

whence we get by inverting that

$$
s \bar{V}(s, x, u, \mu)=\sum_{r=0}^{x} \bar{G}(s, x-r, u, v, \mu) p_{r}^{+}(s), \quad x \geq 0 .
$$


Assuming that the process is lower continuous and using the moment generating transforms

$$
\tilde{\mathcal{G}}(s, \beta, u, v, \mu)=\sum_{x=0}^{\infty} \beta^{x} \bar{G}(s, x, u, v, \mu)
$$

one can derive analogs of Corollaries 1 and 4-6 from Theorem 3.

\section{BIBLIOGRAPHY}

1. D. V. Gusak, On the joint distribution of the first exit time and exit value for homogeneous processes with independent increments, Teor. Veroyatnost. i Primenen. 14 (1969), no. 1, 15-23; English transl. in Theor. Probab. Appl. 14 (1970), no. 1. MR 39:6395

2. Distribution of overjump functionals of a homogeneous process with independent increments, Ukrain. Mat. Zh. 54 (2002), no. 3, 303-322; English transl., Ukrain. Math. J. 54 (2003), no. 3, 371-397. MR 2003j:60067

3. N. S. Bratiı̌chuk and D. V. Gusak, Boundary Problems for Processes with Independent Increments, "Naukova Dumka", Kiev, 1990. (Russian) MR 91m:60139

4. D. V. Gusak and A. I. Tureniyazova, The distribution of some limit functionals for lattice Poisson processes defined on a Markov chain, Asymptotic Methods in Studies of Stochastic Models, Institute of Mathematics, Academy of Sciences of Ukrainian SSR, Kiev, 1987, pp. 2127. (Russian) MR 90f:60132

5. — On lattice semicontinuous processes defined on a Markov chain, Ukrain. Mat. Zh. 39 (1987), no. 6, 707-711; English transl. in Ukrain. Math. J. 39 (1988), no. 6. MR 89a:60172

6. D. V. Gusak, On a generalized semicontinuous integer-valued Poisson process with reflection, Teoriya Imovir. ta Matem. Statist. 59 (1998), 41-46; English transl., Theor. Probability and Math. Statist. 59 (1999), 41-46.

7. $\ldots$, The factorization method in boundary problems for homogeneous processes with independent increments, Distribution of Some Functionals for Processes with Independent Increments, Preprint 85-43, Institute of Mathematics, Academy of Sciences of Ukrainian SSR, Kiev, 1985, pp. 3-42. (Russian)

Institute of Mathematics, National Academy of Sciences of Ukraine, Tereshchenkivs'ka Street 3, Kyiv 01601, Ukraine

E-mail address: random@imath.kiev.ua

Received 18/FEB/2002

Translated by V. SEMENOV 